\title{
AN AGGREGATION-BASED DOMAIN DECOMPOSITION PRECONDITIONER FOR GROUNDWATER FLOW *
}

\author{
E. W. JENKINS ${ }^{\dagger}$, C. T. KELLEY ${ }^{\dagger}$, C. T. MILLER ${ }^{\ddagger}$, AND $\quad$ C. E. KEES ${ }^{\ddagger}$
}

\begin{abstract}
In this paper we give a convergence analysis of a two-level additive Schwarz method in which the coarse mesh basis is constructed with aggregation, a method common in algebraic multigrid. This coarse mesh does not need geometric information about the subdomains and can readily be used on unstructured spatial meshes. We illustrate the performance with several computational examples.
\end{abstract}

Key words. Domain decomposition, Newton-Krylov-Schwarz methods, Richards' equation, Multiphase flow, nonlinear equations,

AMS subject classifications. 65H10, 65N55, 76S05, 76T05, 86A05

1. Introduction. This paper provides a convergence theory for the two-level Schwarz preconditioner first described in [8-10]. The preconditioner is a two-level additive Schwarz method $[4,5,18]$. Its novel feature is the use of aggregation ideas from algebraic multigrid [1,22] and balancing Neumann-Neumann methods [13-15] to construct the coarse mesh. The use of aggregation arose from necessity. In the applications reported in [8-10] the subdomains were irregular, and a coarse mesh based on "hat functions" over the subdomains was impractical. For the same reason, we needed minimal overlap between subdomains. Unlike the method from [3], we do not need to create a coarse mesh geometry or use geometric information about the subdomains.

While our theoretical results allow for overlap, and some of the experimental results in $\S 3$ are for subdomains with substantial overlap, the application of the preconditioner in practice has been for minimally overlapping subdomains.

Our analysis uses the standard finite element framework from [18,24]. The preconditioner also works well in the context of finite differences, however, as some of the examples in $\S 3$ illustrate.

This preconditioner was developed for use in the Adaptive Hydrology model (ADH) [19], a production code being developed at the US Army Engineer Research and Development Center. Because ADH is a three-dimensional unstructured mesh code, detailed grid refinement studies are too costly to perform; therefore, in this paper we illustrate the performance of the preconditioner with computational examples in two space dimensions, a setting in which grid-refinement studies and investigations of the effects of overlap can more readily be done.

1.1. Problem Formulation. We begin with the weak form of an elliptic boundary value problem on a domain $\Omega \subset R^{d}$ with boundary $\Gamma$. The goal is to find $u \in V$ such that

$$
a(u, v)=l(v) \text { for all } v \in V
$$

*Version of May 15, 2000. This research was supported in part by Army Research Office contract DAAD19-991-0186, US Army contract DACA39-95-K-0098, National Science Foundation grant DMS-9700569, a Cray Research Corporation Fellowship, and a Department of Education GAANN fellowship. Computing activity was partially supported by an allocation from the North Carolina Supercomputing Center.

${ }^{\dagger}$ North Carolina State University, Center for Research in Scientific Computation and Department of Mathematics, Box 8205, Raleigh, N. C. 27695-8205, USA (ewjenkin@eos.ncsu.edu, Tim_Kelley@ncsu.edu,).

$\ddagger$ Department of Environmental Sciences and Engineering, 104 Rosenau Hall, University of North Carolina, Chapel Hill, NC 27599-7400 (casey_miller@unc.edu, chris_kees@unc.edu). 
where $l$ is a linear functional on $V$, and $V$ is an appropriate function space. In our examples the problems will be second order with Dirichlet or Neumann boundary conditions and $V \subset H^{1}(\Omega)$. We take approximating spaces of continuous functions $V^{h} \subset V$. The approximating problem at level $h$ is to find $u^{h} \in V^{h}$ such that

$$
a\left(u^{h}, v\right)=l(v) \text { for all } v \in V^{h} .
$$

The problem, (1.2), is equivalent to a linear system

$$
A u^{h}=f
$$

on $V^{h}$ where $a(u, v)=(A u, v)$ for all $u, v \in V^{h}$. Here $(\cdot, \cdot)$ is the $L^{2}(\Omega)$ inner product.

Schwarz preconditioners are designed to accelerate Krylov space iterative methods for the solution of (1.3).

1.2. One-level Method. We begin with the one-level additive Schwarz preconditioner. We divide $\Omega$ into subdomains $\left\{\Omega_{i}\right\}_{i=1}^{J}$ with an overlap width of at least $\delta$ and assume that $\bigcup_{i=1}^{J} \Omega_{j}=\Omega$.

Let $R_{j}$ be the restriction map from an element of $V^{h}$ to the subspace $V_{j}$ of $V^{h}$ with support on $\Omega_{j}$. Let

$$
A_{j}=R_{j} A R_{j}^{T}
$$

be the subdomain operator. We assume that $A_{j}$ is nonsingular on $V_{j}$ and define

$$
B_{j}=R_{j}^{T} \tilde{A}_{j}^{-1} R_{j},
$$

where $\tilde{A}_{j}$ is an approximation of $A_{j}$. The one-level additive Schwarz preconditioner is

$$
M=\sum_{j=1}^{J} B_{j} .
$$

1.3. Two-level Method. The two-level additive Schwarz method adds a coarse mesh term

$$
B_{0}=R_{0}^{T} \tilde{A}_{0}^{-1} R_{0}
$$

to the one-level preconditioner. Here $\tilde{A}_{0}$ is an approximation of $A_{0}$. We let $V^{H}$ denote the space of coarse mesh basis functions. If the coarse mesh restriction map $R_{0}$ and the coarse mesh operator $A_{0}$ are well designed, the condition number of $M A$ is significantly reduced.

One way to define a coarse mesh problem is to discretize the continuous problem on a coarser mesh. There are a few difficulties associated with forming the coarse problem this way. For unstructured meshes, such as the ones considered in [8-10], the interpolation operators between the fine mesh and the coarse mesh are difficult to define. Second, a coarse mesh must be generated, stored, and parallelized. Finally, the PDE must be discretized and recomputed on the coarse mesh.

Alternatively, the discretization of the coarse mesh operator may be defined in terms of the existing fine mesh discretization. A Galerkin or variational coarse grid correction uses the fine grid matrix to obtain the coarse grid operator as $A_{0}=R_{0} A R_{0}^{T}$, where $R_{0}$ is the interpolation operator to the coarse mesh function space, and $R_{0}^{T}$ is the restriction operator. Methods that obtain the coarse mesh matrix by using the fine mesh matrix are called nested or multilevel methods [18]. If the 
coarse mesh basis functions are obtained from the fine mesh basis functions, then the coarse mesh space $V^{H}$ is contained in the fine grid space $V^{h}$.

We use the aggregation-based basis from [8-10] in this paper, where one coarse mesh basis function is defined for each subdomain as the sum of the fine mesh basis functions for that subdomain.

To set the notation that we will need in $\S 2$, let the expansion of a function $u \in V^{h}$ in the finite element basis be

$$
u=\sum_{i} u_{i} \psi_{i}
$$

where the $\psi_{i}$ 's are the basis functions for the fine mesh, and a function $u_{C} \in V^{H}$ can be represented on the coarse mesh space as

$$
u_{C}=\sum_{K} u_{C_{K}} \Psi_{k}
$$

where the $\Psi_{K}$ 's are the finite element basis functions for the coarse mesh space. Since $V^{H} \subset V^{h}$, $\Psi_{K}$ can be written as

$$
\Psi_{K}=\sum_{j} R_{K j} \psi_{j}
$$

The index $K$ represents the subdomain number. For our coarse mesh basis functions, the value of $R_{K j}$ is either 0 or 1 . If the fine mesh basis function $\psi_{j}$ is contained in subdomain $K$, then $R_{K j}=1$; otherwise, $R_{K j}=0$.

Further expanding the representation of $u_{C}$ gives

$$
\begin{aligned}
u_{C} & =\sum_{K} u_{C_{K}} \Psi_{K} \\
& =\sum_{K} u_{C_{K}} \sum_{j} R_{K j} \psi_{j} \\
& =\sum_{j}\left(\sum_{K} u_{C_{K}} R_{K j}\right) \psi_{j} \\
& =\sum_{j}\left(R^{T} u_{C}\right)_{j} \psi_{j}
\end{aligned}
$$

Thus $R^{T}$ is the operator which interpolates from the coarse mesh to the fine mesh. Any function on the coarse mesh can be represented solely in terms of the already existing fine mesh functions, making the formulation of a separate coarse mesh unnecessary.

1.4. Condition Estimate. In Assumption 1.1 we make precise the idea that $H$ is the characteristic diameter of a subdomain. In Assumption 1.2 we make precise the overlap $\delta$ between the subdomains and the properties of the coarse mesh basis functions.

ASSUMPTION 1.1.

1. There is $C>0$ such that $\operatorname{diam}\left(\Omega_{j}\right) \leq C H$ for all $j=1, \ldots, J$.

2. There is $c>0$ such that for all $x \in \Omega$ there exists $j \geq 1$ such that $x \in \Omega_{j}$ and

$$
\operatorname{dist}\left(x, \partial \Omega_{j} \backslash \partial \Omega\right) \geq c \delta .
$$


3. There are $C_{R}, C_{1}, C_{2}>0$ such that for all $x \in \Omega$ the ball

$$
B\left(x, C_{R} H\right)=\left\{y \in \Omega: \operatorname{dist}(y, x) \leq C_{R} H\right\}
$$

intersects at most $C_{1}+C_{2}^{d}$ subdomains $\Omega_{j}$ (i.e., an object of diameter $O(H)$ intersects at most $O(1)$ subdomains $\Omega_{i}$ ).

4. $\mu\left(\Omega_{j}\right) \geq C H^{d}, j=1, \ldots, J$.

In Assumption 1.1, $\mu$ denotes Lebesgue measure.

ASSUMPTION 1.2. Assume the basis functions $\Psi_{i}$ of the coarse space satisfy

1. $\left|\Psi_{i}\right|_{H^{1}(\Omega)}^{2} \leq C \frac{H^{d-1}}{\delta}$

$\left\|\Psi_{i}\right\|_{L^{2}}^{2} \leq C H^{d}$

2. There is a domain $\Omega^{\text {int }} \subset \Omega$ such that $\sum_{i} \Psi_{i}(x)=1$ for every $x \in \Omega^{\text {int }}$ and $\operatorname{dist}(x, \partial \Omega) \leq$ $C \delta$ for every $x \in \Omega \backslash \Omega^{i n t}$.

3. $\operatorname{supp}\left(\Psi_{i}\right) \subset \bar{\Omega}_{i}$.

Parts 1 and 2 of Assumption 1.2 differ from similar assumptions in [1] in the $H^{1}$ semi-norm of the coarse mesh basis functions, and in the distance from the space $\Omega^{i n t}$ to $\partial \Omega$.

In $\S 2$ we prove

THEOREM 1.1. Let $V^{h}=\sum_{j=0}^{J} V_{j} \subset C(\bar{\Omega})$ and let Assumptions 1.1 and 1.2 hold. Assume that there is $\omega \geq 1$ such that

$$
(v, v) \leq\left(\tilde{A}_{j}^{-1} A_{j} v, v\right) \leq \omega(v, v)
$$

for all $v \in V^{h}$ and $0 \leq j \leq J$. Let

$$
M=\sum_{j=0}^{J} B_{j}
$$

then there is $C>0$, independent of $H$ and $h$ such that

$$
\kappa(M A) \leq C \omega\left(1+(H / h)^{2}\right) .
$$

2. Convergence Theory. We base our analysis on the result from [23, 24].

THEOREM 2.1. Let $K_{0}$ be a positive constant so that, for any $v \in V^{h}$, there exists a decomposition $v=\sum_{i=0}^{J} v_{i}$ such that $v_{i} \in V_{i}$ and

$$
\sum_{i=0}^{J}\left(A_{i} v_{i}, v_{i}\right) \leq K_{0}(A v, v)
$$

Let

$$
K_{1}=\max _{1 \leq j \leq J} \sum_{i=1}^{J} \varepsilon_{i j},
$$

where, for $1 \leq i, j \leq J, \varepsilon_{i j}=0$ if $P_{i} P_{j}=0$ (i.e., if $V_{i} \perp V_{j}$ ), $\varepsilon_{i j}=1$ otherwise. Then

$$
\kappa(M A) \leq \omega K_{0}\left(1+K_{1}\right),
$$


where

$$
\omega=\max _{0 \leq j \leq J} \lambda_{\max }\left(\tilde{A}_{j}^{-1} A_{j}\right) .
$$

We assume that the energy norm is equivalent to the $H^{1}$ seminorm, and we can therefore replace (2.1) by

$$
\sum_{i=0}^{J}\left|u_{i}\right|_{H^{1}(\Omega)}^{2} \leq K_{0}|u|_{H^{1}(\Omega)}^{2} .
$$

Our estimate for $K_{0}$ will be based on (2.4).

The value of $K_{1}$ is an indicator of the number of subdomains that contain any given point in $\Omega$; we assume our partition is such that $K_{1}=O(1)$. We solve the subdomain problems exactly in our numerical results, so $\omega=1$ in $\S 3$. Thus our condition number estimate is based on the estimate of $K_{0}$, which we obtain using Lemmas 2.2 and 2.3. Our analysis uses some of the techniques developed in [1], but we do not smooth the coarse mesh basis. Our estimates in Lemma 2.2 are thus different from those in [1].

We define the coarse mesh operator $Q: V^{h} \rightarrow V^{H}$ by

$$
Q u=\sum_{i=1}^{J} \alpha_{i} \Psi_{i}, \quad \alpha_{i}=\alpha_{i}(u)=\frac{1}{\mu\left(\Omega_{i}\right)} \int_{\Omega_{i}} u(x) d x,
$$

where $u \in V^{h}$. Using the Cauchy-Schwarz inequality and Assumption 1.1 we get

$$
\left|\int_{\Omega_{i}} u(x) d x\right| \leq C\|u\|_{L^{2}} H^{d / 2} .
$$

Hence

$$
\left|\alpha_{i}\right| \leq C \frac{H^{d / 2}}{H^{d}}\|u\|_{L^{2}}=C H^{-d / 2}\|u\|_{L^{2}} .
$$

The value of $K_{0}$ depends on the bound of the energy of $Q u$ and on the $L^{2}$ bound of the error in the coarse mesh operator, i.e., $u-Q u$. These bounds are provided in Lemma 2.2.

Here, as in the remainder of this section, $C$ is a constant that is independent of $H$ and $h$. $C$ may increase as the analysis progresses.

LEMMA 2.2. If Assumptions 1.1 and 1.2 hold, then

$$
\begin{aligned}
\|u-Q u\|_{L^{2}}^{2} & \leq C H^{2}|u|_{H^{1}}^{2} \\
|Q u|_{H^{1}}^{2} & \leq C \frac{H}{\delta}|u|_{H^{1}}^{2} .
\end{aligned}
$$

Proof. Let

$$
\begin{aligned}
B & =\Omega \backslash \Omega^{i n t} \\
\mathcal{B} & =\left\{i: \Omega_{i} \cap B \neq \emptyset\right\} \\
B^{\prime} & =\bigcup_{i \in \mathcal{B}} \Omega_{i} \\
W & =\sup _{x \in B^{\prime}}\{\operatorname{dist}(x, \partial \Omega)\} \\
B_{0} & =\{x \in \Omega: \operatorname{dist}(x, \partial \Omega) \leq W\} .
\end{aligned}
$$


If $x \in B$, then $\operatorname{dist}(x, \partial \Omega) \leq C \delta$ by Assumption 1.2. Thus

$$
B^{\prime}=\cup_{i}\left\{\Omega_{i}: \partial \Omega_{i} \cap \partial \Omega \neq \emptyset\right\} .
$$

Therefore, we have that $\operatorname{dist}_{x \in B^{\prime}}(x, \partial \Omega) \leq C H$ since $\operatorname{diam}\left(\Omega_{i}\right) \leq C H$ for all $i$. Hence $W \leq C H$ and using the Poincaré inequality [6] we get

$$
\|u\|_{L^{2}(B)} \leq\|u\|_{L^{2}\left(B_{0}\right)} \leq C H|u|_{H^{1}\left(B_{0}\right)} .
$$

Let $\mathcal{N}_{i}=\left\{j: \Omega_{j} \cap \Omega_{i} \neq \emptyset\right\}$ and let $\operatorname{card}\left(\mathcal{N}_{i}\right)$ denote the cardinality of $\mathcal{N}_{i}$. We have

$$
\begin{aligned}
\|Q u\|_{L^{2}(B)}^{2} & =\left(\sum_{i \in \mathcal{B}} \alpha_{i}(u) \Psi_{i}(u), \sum_{j \in \mathcal{B}} \alpha_{j}(u) \Psi_{j}(u)\right) \\
& \leq \sum_{i \in \mathcal{B}} \sum_{j \in \mathcal{N}_{i} \cap \mathcal{B}}\left|\alpha_{i}(u)\right|\left|\alpha_{j}(u)\right|\left\|\Psi_{i}\right\|_{L^{2}}\left\|\Psi_{j}\right\|_{L^{2}} \\
& \leq \frac{1}{2} \sum_{i \in \mathcal{B}} \sum_{j \in \mathcal{N}_{i} \cap \mathcal{B}}\left(\alpha_{i}^{2}(u)+\alpha_{j}^{2}(u)\right) C H^{d} \quad \text { since } a^{2}+b^{2} \geq 2 a b \\
& \leq C H^{d} \max \left\{\operatorname{card}\left(N_{i}\right)\right\} \sum_{i \in \mathcal{B}} \alpha_{i}^{2}(u) \leq C \sum_{i \in \mathcal{B}}\|u\|_{L^{2}\left(\Omega_{i}\right)}^{2} \leq C\|u\|_{L^{2}\left(B_{0}\right)}^{2} .
\end{aligned}
$$

Therefore

$$
\|u-Q u\|_{L^{2}(B)} \leq\|u\|_{L^{2}(B)}+\|Q u\|_{L^{2}(B)} \leq C\|u\|_{L^{2}(B)} \leq C H|u|_{H^{1}(B)} .
$$

Since $\left|\Psi_{i}\right|_{H^{1}}^{2} \leq C \frac{H^{d-1}}{\delta}$ we have

$$
\begin{aligned}
|Q u|_{H^{1}(B)}^{2} & \leq C \frac{H^{d-1}}{\delta} \max \left\{\operatorname{card}\left(\mathcal{N}_{i}\right)\right\} \sum_{i \in \mathcal{B}} \alpha_{i}^{2}(u) \\
& \leq C \frac{1}{H \delta} \sum_{i \in \mathcal{B}}\|u\|_{L^{2}\left(\Omega_{i}\right)}^{2} \leq C \frac{1}{H \delta}\|u\|_{L^{2}\left(B_{0}\right)}^{2} \\
& \leq C \frac{1}{H \delta} H^{2}|u|_{H^{1}\left(B_{0}\right)}^{2}=C \frac{H}{\delta}|u|_{H^{1}\left(B_{0}\right)}^{2} .
\end{aligned}
$$

The estimates above are for the set $B$ around the edge of the domain. We complete the proof by making similar estimates in $\Omega^{\text {int }}$.

Let $u_{E}$ be an extension of $u$ such that $u_{E}=u$ on $\Omega$ and

$$
\left|u_{E}\right|_{H^{1}\left(\mathcal{R}^{d}\right)} \leq C(\Omega)|u|_{H^{1}(\Omega)} .
$$

Let $B_{i}^{\prime}=\bigcup_{j \in \mathcal{N}_{i}} \Omega_{j}$, and let $B_{i}$ be the ball around $B_{i}^{\prime}$. Then by Assumption 1(c), we have that $\operatorname{diam}\left(B_{i}\right) \leq C H$.

For $j=1, \ldots, J$, define $c_{j}=\frac{1}{\mu\left(B_{j}\right)} \int_{B_{j}} u_{E} d x, \bar{u}_{j}=u_{E}-c_{j}$. Assumption 1.2 and the definition of $Q$ imply that for $x \in \Omega \cap \Omega^{i n t}$,

$$
Q u(x)=Q \bar{u}_{i}(x)+c_{i} .
$$


Therefore

$$
\begin{aligned}
\|u-Q u\|_{L^{2}\left(\Omega^{i n t}\right)}^{2} & \leq \sum_{i=1}^{J}\|u-Q u\|_{L^{2}\left(\Omega_{i} \cap \Omega^{i n t}\right)}^{2} \\
& =\sum_{i=1}^{J}\left\|\bar{u}_{i}+c_{i}-Q\left(\bar{u}_{i}+c_{i}\right)\right\|_{L^{2}\left(\Omega_{i} \cap \Omega^{i n t}\right)}^{2} \\
& =\sum_{i=1}^{J}\left\|\bar{u}_{i}-Q \bar{u}_{i}\right\|_{L^{2}\left(\Omega_{i} \cap \Omega^{i n t}\right)}^{2} \quad \text { since } \quad Q c_{i}=c_{i} \\
& \leq \sum_{i=1}^{J}\left\|\bar{u}_{i}\right\|_{L^{2}\left(B_{i}\right)}^{2}+\left\|Q \bar{u}_{i}\right\|_{L^{2}\left(\Omega_{i} \cap \Omega^{i n t}\right)}^{2} \cdot
\end{aligned}
$$

We use

$$
\begin{aligned}
\left\|Q \bar{u}_{i}\right\|_{L^{2}\left(\Omega_{i} \cap \Omega^{i n t}\right)}^{2} & \leq\left\|\sum_{j \in \mathcal{N}_{i}} \alpha_{j}\left(\bar{u}_{i}\right) \Psi_{j}\right\|_{L^{2}(\Omega)}^{2} \leq\left(\sum_{j \in \mathcal{N}_{i}}\left|\alpha_{j}\left(\bar{u}_{i}\right)\right|\left\|\Psi_{j}\right\|_{L^{2}\left(\Omega_{j}\right)}\right)^{2} \\
& \leq \operatorname{card}\left(\mathcal{N}_{i}\right) \sum_{j \in \mathcal{N}_{i}} \alpha_{j}^{2}\left(\bar{u}_{i}\right)\left\|\Psi_{j}\right\|_{L^{2}\left(\Omega_{j}\right)}^{2} \leq C H^{d} \sum_{j \in \mathcal{N}_{i}} \alpha_{j}^{2}\left(\bar{u}_{i}\right) \\
& \leq C H^{d} H^{-d} \sum_{j \in \mathcal{N}_{i}}\left\|\bar{u}_{i}\right\|_{L^{2}\left(\Omega_{j}\right)}^{2} \leq C\left\|\bar{u}_{i}\right\|_{L^{2}\left(B_{i}\right)}^{2},
\end{aligned}
$$

and the Poincaré inequality to obtain

$$
\begin{aligned}
\|u-Q u\|_{L^{2}\left(\Omega^{i n t}\right)}^{2} & \leq 2 \sum_{i=1}^{J}\left\|\bar{u}_{i}\right\|_{L^{2}\left(B_{i}\right)}^{2} \leq C H^{2} \sum_{i=1}^{J}\left|\bar{u}_{i}\right|_{H^{1}\left(B_{i}\right)}^{2} \\
& =C H^{2} \sum_{i=1}^{J}\left|u_{E}\right|_{H^{1}\left(B_{i}\right)}^{2} \leq C H^{2} \sum_{i=1}^{J}|u|_{H^{1}\left(B_{i}\right)}^{2} \leq C H^{2}|u|_{H^{1}(\Omega)}^{2} .
\end{aligned}
$$

This and (2.8) imply (2.6).

We complete the proof of (2.5) by combining (2.9) with the estimate

$$
\begin{aligned}
|Q u|_{H^{1}\left(\Omega^{i n t}\right)}^{2} & \leq \sum_{i=1}^{J}|Q u|_{H^{1}\left(\Omega_{i} \cap \Omega^{i n t}\right)}^{2}=\sum_{i=1}^{J}\left|Q\left(\bar{u}_{i}+c_{i}\right)\right|_{H^{1}\left(\Omega_{i} \cap \Omega^{i n t}\right)}^{2} \\
& =\sum_{i=1}^{J}\left|Q \bar{u}_{i}+c_{i}\right|_{H^{1}\left(\Omega_{i} \cap \Omega^{i n t}\right)}^{2}=\sum_{i=1}^{J}\left|Q \bar{u}_{i}\right|_{H^{1}\left(\Omega_{i} \cap \Omega^{i n t}\right)}^{2} \\
& \leq \sum_{i=1}^{J}\left|\sum_{j \in \mathcal{N}_{i}} \alpha_{j}\left(\bar{u}_{i}\right) \Psi_{j}\right|_{H^{1}(\Omega)}^{2} \leq \sum_{i=1}^{J} \operatorname{card}\left(\mathcal{N}_{i}\right) \sum_{j \in \mathcal{N}_{i}} \alpha_{j}^{2}\left(\bar{u}_{i}\right)\left|\Psi_{j}\right|_{H^{1}(\Omega)}^{2} \\
& \leq C \frac{1}{H^{d}} \frac{H^{d-1}}{\delta} \sum_{i=1}^{J} \sum_{j \in \mathcal{N}_{i}}\left\|\left.\bar{u}_{i}\right|_{L^{2}\left(\Omega_{j}\right)} ^{2} \leq C \frac{1}{H \delta} \sum_{i=1}^{J}\right\| \bar{u}_{i} \|_{L^{2}\left(B_{i}\right)}^{2} \\
& \leq C \frac{1}{H \delta} H^{2} \sum_{i=1}^{J}\left|\bar{u}_{i}\right|_{H^{1}\left(B_{i}\right)}^{2}=C \frac{H}{\delta} \sum_{i=1}^{J}\left|u_{E}\right|_{H^{1}\left(B_{i}\right)}^{2} \leq C \frac{H}{\delta}|u|_{H^{1}(\Omega)}^{2} .
\end{aligned}
$$

This completes the proof. 
LEMMA 2.3. Under Assumptions 1.1 and 1.2, for every finite element function $u \in V^{h}$, there exists a decomposition $\left\{u_{i}\right\}_{i=0}^{J}, u_{i} \in V_{i}$, such that

$$
\begin{aligned}
u & =\sum_{i=0}^{J} u_{i} \\
\sum_{i=0}^{J}\left|u_{i}\right|_{H^{1}(\Omega)}^{2} & \leq C\left(1+\frac{H}{\delta}\right)^{2}|u|_{H^{1}(\Omega)}^{2} .
\end{aligned}
$$

Proof. Define $I_{h}$ to be the fine mesh operator $I_{h}: \mathcal{C} \rightarrow V_{h}$ by

$$
I_{h}(u)=\sum_{i=1}^{n} u\left(x_{i}\right) \psi_{i}
$$

where $\left\{\psi_{i}\right\}_{i=1}^{n}$ is the finite element basis on the fine mesh, and $\left\{x_{i}\right\}_{i=1}^{n}$ are the fine mesh nodal points. Let $u$ be partitioned such that

$$
u_{0}=Q u \text { and } u_{i}=I_{h}\left(\theta_{i}(u-Q u)\right),
$$

where $\left\{\theta_{i}\right\}$ is a partition of unity such that $\theta_{i}(x)=1$ for $x \in \Omega_{i}^{i n t}, \theta_{i}=0$ for $x \notin \Omega_{i}$, and $\left|\nabla \theta_{i}\right| \leq \frac{1}{\delta}$. Clearly, by construction,

$$
\sum_{i=0}^{J} u_{i}=u
$$

The standard arguments [18] imply that

$$
\sum_{i=0}^{J}\left|u_{i}\right|_{H^{1}(\Omega)}^{2} \leq C\left(\frac{1}{\delta^{2}}\|u-Q u\|_{L^{2}(\Omega)}^{2}+|u-Q u|_{H^{1}(\Omega)}^{2}+|Q u|_{H^{1}(\Omega)}^{2}\right)
$$

We complete the proof by applying Lemma 2.2 to estimate $|Q u|_{H^{1}(\Omega)}^{2}$ and $\|u-Q u\|_{L^{2}(\Omega)}^{2}$.

If we let

$$
K_{0}=C\left(1+\frac{H}{\delta}\right)^{2}
$$

we complete the proof of Theorem 1.1.

\section{Numerical Results.}

3.1. Laplace Equation. In this section we consider the simple test problem

$$
\nabla^{2} u=0
$$

on the unit square $[0,1] \times[0,1]$ with zero Dirichlet boundary conditions. In both $\S 3.1 .1$ and 3.1.2 we use the function identically equal to one on the mesh as the initial iterate for a preconditioned conjugate gradient iteration. We terminated the iterations when the residual had been reduced by a factor of $10^{-4}$.

We obtained similar results using GMRES [17], Bi-CGSTAB [20], and TFQMR [7] in the tests. 
3.1.1. Finite Difference Discretizations with Overlap. The experiments in this section were designed to measure the effects of overlap. We let $h=2^{-m}$ be the scale of the fine mesh and let the overlap $o$ be the is the nearest integer larger than

$$
2^{m} o_{1}+o_{0}
$$

where $o_{1}$ is the overlap that depends on the physical domain, and $o_{0}$ is the overlap that is a constant number of gridlines. The grid is an $n \times n$ mesh where $n=2^{m}+o$. In this way we can subdivide the region into $4^{p}$ subdomains, each of size $m \times m$, where

$$
m=2^{m-p}+o-1
$$

The scale $H$ of the subdomains is $2^{-p}$.

We considered overlaps of $1\left(o_{0}=1, o_{1}=0\right)$ and $1 \%\left(o_{0}=0, o_{1}=.01\right)$. We discretized with five point differences and use the function identically one on the mesh as the initial iterate. We used the MATLAB software associated with [12] for these experiments and used bilinear coarse mesh basis functions. These do not form a partition of unity as a piecewise linear set would and were used to illustrate the flexibility of the preconditioner.

In Table 3.1 we tabulate the number of conjugate gradient iterations needed for termination for several values of $h$ and $H$, with an overlap of 1 and $1 \%$. As the theory predicts, the iteration count is constant as $h$ and $H$ are reduced simultaneously for the overlap of 1 and declines as $H$ is reduced with the overlap of $1 \%$.

TABLE 3.1

Iteration Statistics for Two-level Additive Schwarz with CG

\begin{tabular}{l|rrrr|rrrr}
\hline & \multicolumn{4}{|c|}{ Overlap = 1 } & \multicolumn{4}{|c}{ Overlap = 1\% } \\
\hline$H \backslash h$ & $1 / 32$ & $1 / 64$ & $1 / 128$ & $1 / 256$ & $1 / 32$ & $1 / 64$ & $1 / 128$ & $1 / 256$ \\
\hline $1 / 4$ & 12 & 16 & 21 & 28 & 12 & 16 & 17 & 20 \\
$1 / 8$ & 12 & 15 & 21 & 27 & 12 & 15 & 17 & 19 \\
$1 / 16$ & 8 & 12 & 16 & 21 & & 12 & 13 & 15 \\
$1 / 32$ & & 8 & 12 & 15 & & & 10 & 11 \\
$1 / 64$ & & & 8 & 12 & & & & 8 \\
$1 / 128$ & & & & 8 & & & & \\
\hline
\end{tabular}


3.1.2. Finite Element Discretization with Minimal Overlap. In Table 3.2 we report similar results for a piecewise linear finite element discretization of (3.1). The overlap in this computation was minimal.

TABLE 3.2

Finite Element Discretization

\begin{tabular}{c|ccc}
\hline $\mathrm{H} \backslash \mathrm{h}$ & $1 / 64$ & $1 / 128$ & $1 / 256$ \\
\hline $1 / 4$ & 37 & 51 & 68 \\
$1 / 8$ & 32 & 44 & 61 \\
$1 / 16$ & 26 & 36 & 49 \\
$1 / 32$ & & 26 & 37 \\
\hline
\end{tabular}

3.2. Richards' Equation Results. In this section we consider a test problem that models groundwater flow in a partially saturated, homogeneous soil. The model equation is the "headbased" form of Richards' equation,

$$
\left[\frac{\partial \theta}{\partial \psi}+\frac{S_{s}}{\theta_{s}} \theta\right] \frac{\partial \psi}{\partial t}=\nabla \cdot\left[K_{s} k_{r} \nabla(\psi+z)\right],
$$

where $\psi$ is the pressure head, $\theta$ is volume fraction of the wetting phase, and $k_{r}$ is the relative permeability of the wetting phase. The remaining terms are scalar coefficients given in Table 3.3, along with their values for the test problem. $\theta$ and $k_{r}$ are functions of $\psi$ given by,

$$
\begin{aligned}
\theta & =\left(\theta_{s}-\theta_{r}\right)\left(1+|\alpha \hat{\psi}|^{n}\right)^{-m}+\theta_{r} \\
k_{r} & =\left(1+|\alpha \hat{\psi}|^{n}\right)^{-m / 2}\left[1-|\alpha \hat{\psi}|^{n-1}\left(1+|\alpha \hat{\psi}|^{n}\right)^{-m}\right]^{2} \\
\hat{\psi} & =\min (\psi, 0),
\end{aligned}
$$

where $m=1-1 / n$. These functions are derived from the van Genuchten [21] and Mualem [16] empirical relations among pressure, saturation, and relative permeability.

The test domain is the unit square $[0,1 \mathrm{~m}] \times[0,1 \mathrm{~m}]$ with boundary and initial conditions,

$$
\begin{aligned}
\psi(x, 0) & =0.0, & x \in[0,1] & & t>0 \\
\psi(x, 1) & =0.1, & x \in[1 / 3,2 / 3] & & t>0 \\
\frac{\partial \psi}{\partial z}(x, 1) & =-1.0, & x \in[0,1 / 3) \cup(2 / 3,1] & & t>0 \\
\frac{\partial \psi}{\partial x}(x, z) & =0.0, & x=0,1 \quad z \in[0,1] & & t>0 \\
\psi(x, z) & =-z, & x, z \in[0,1] \times[0,1] & & t=0
\end{aligned}
$$

3.2.1. Finite Difference Discretization with Minimal Overlap. We discretized equation 3.3 by applying cell-centered finite differences to the spatial operator, thereby yielding the system of 
TABLE 3.3

Richards' equation parameters

\begin{tabular}{|l|l|l|}
\hline Description & Symbol & Value \\
\hline Saturated volume fraction & $\theta_{s}$ & $3.01 \times 10^{-1}$ \\
Residual volume fraction & $\theta_{r}$ & $9.30 \times 10^{-2}$ \\
Specific storage & $S_{s}$ & $1.00 \times 10^{-6}(1 / \mathrm{m})$ \\
Hydraulic conductivity & $K_{s}$ & $5.04 \times 10^{0}(\mathrm{~m} /$ day $)$ \\
Mean pore size & $\alpha$ & $5.47 \times 10^{0}(1 / \mathrm{m})$ \\
Pore size uniformity & $n$ & $4.26 \times 10^{0}$ \\
\hline
\end{tabular}

differential-algebraic equations,

$$
\begin{aligned}
F_{i, j}\left(t, \psi, \frac{\partial \psi}{\partial t}\right)= & \left(\frac{d \theta}{d \psi}{ }_{i, j}+\frac{S_{s}}{\theta_{s}} \theta_{i, j}\right) \frac{\partial \psi_{i, j}}{\partial t} \\
& -\frac{1}{\Delta z^{2}}\left[K_{i+\frac{1}{2}, j}\left(\psi_{i+1, j}-\psi_{i, j}\right)-K_{i-\frac{1}{2}, j}\left(\psi_{i, j}-\psi_{i-1, j}\right)\right] \\
& -\frac{1}{\Delta z}\left(K_{i+\frac{1}{2}, j}-K_{i-\frac{1}{2}, j}\right) \\
& -\frac{1}{\Delta x^{2}}\left[K_{i, j+\frac{1}{2}}\left(\psi_{i, j+1}-\psi_{i, j}\right)-K_{i, j-\frac{1}{2}}\left(\psi_{i, j}-\psi_{i, j-1}\right)\right]
\end{aligned}
$$

where $i=0, \ldots, N-1, j=0, \ldots, N-1, \Delta z=\Delta x=1 / N$, and

$$
\begin{aligned}
& K_{i \pm \frac{1}{2}, j}=\left[\left(K_{s} k_{r}\right)_{i \pm 1, j}+\left(K_{s} k_{r}\right)_{i, j}\right] / 2 \\
& K_{i, j \pm \frac{1}{2}}=\left[\left(K_{s} k_{r}\right)_{i, j \pm 1}+\left(K_{s} k_{r}\right)_{i, j}\right] / 2
\end{aligned}
$$

The semidiscrete system was integrated in time over [0, 0.0149 days] by applying the fixed leading coefficient backward difference formulas of orders one to five $[2,11]$. Order and step-size were selected via local truncation error estimates, and the local truncation error tolerance was set to $10 / \Delta x^{2}$.

At a given step, $t_{n+1}$, the application of the integration method yielded a nonlinear system of the form,

$$
F\left[t_{n+1}, \psi_{n+1}, g\left(\psi_{n+1}\right)\right]=G\left(\psi_{n+1}\right)=0
$$

where $g(y)$ is a the backward difference formula for $\partial \psi / \partial t$. We solved the nonlinear system with an inexact Newton iteration that terminated when the 2-norm of the nonlinear residual was reduced by a factor of $10^{-5}$.

At each Newton iteration we obtained the Newton step, $\delta^{m+1}$, by solving the linear system,

$$
\left[\frac{\partial G}{\partial \psi}\left(\psi_{n+1}^{m}\right)\right] \delta^{m+1}=-G\left(\psi_{n+1}^{m}\right),
$$

with scaled, preconditioned, BiCGstab. The scaling was obtained from the integration method's weighted root mean squared norm. Such a scaling would, in real applications, allow termination of the linear iteration according to tolerances specified by the integration scheme in real applications; however, for this test we iterated until the 2-norm of the true linear residual was reduced by a 
TABLE 3.4

Richards' Equation Iteration Statistics

\begin{tabular}{c|ccccc}
\hline$H \backslash h$ & $1 / 16$ & $1 / 32$ & $1 / 64$ & $1 / 128$ & $1 / 256$ \\
\hline $1 / 8$ & 7 & 8 & 9 & 12 & 15 \\
$1 / 16$ & & 7 & 9 & 11 & 14 \\
$1 / 32$ & & & 7 & 9 & 11 \\
$1 / 64$ & & & & 7 & 9 \\
$1 / 128$ & & & & & 7 \\
\hline
\end{tabular}

factor of $10^{-7}$ to insure that errors in the Newton step were insignificant with respect to the Newton iteration and integration.

The preconditioner was two-level additive Schwarz with the coarse grid correction determined from the restriction and interpolation operators in $\S 1.3$. The subdomains had the minimal overlap of $\Delta x=1 / N$. Table 3.4 gives the average BiCGstab iterations per Newton iteration for two-level additive Schwarz. The iteration count is constant as $H$ and $h$ are reduced simultaneously, which is consistent with the predictions of the theory.

Acknowledgments. The authors wish to thank Marian Brezina, Jim Jones, Van Henson, David Keyes, Andrea Toselli, Mary Wheeler, Carol Woodward, and Jun Zou for many helpful discussions and Dave Klepacki, Matt Matthews, Mark Reed, Eric Sills, and Bob Walkup, for their help with the computations. We are particularly grateful to our collaborators at the US Army Engineer Research and Development Center: Charlie Berger, Jackie Hallberg, Stacy Howington, John Peters, Joe Schmidt, and Alan Stagg.

\section{REFERENCES}

[1] M. BREZINA, Robust Iterative Methods on Unstructured Meshes, PhD thesis, University of Colorado at Denver, Denver, Colorado, 1997.

[2] P. N. Brown, A. C. Hindmarsh, And L. R. Petzold, Using Krylov methods in the solution of large-scale differential-algebraic systems, SIAM J. Sci. Comput., 15 (1994), pp. 1467-1488.

[3] T. F. ChAN, S. Go, AND J. Zou, Boundary treatments for multilevel methods on unstructured grids, SIAM J. Sci. Comput., 21 (1999), pp. 46-66.

[4] M. DRYJA AND O. B. WidLUnD, An additive variant of the Schwarz alternating method for the case of many subregions, Tech. Rep. 339, Department of Computer Science, Courant Institute, 1987.

[5] _ Some domain decomposition algorithms for elliptic problems, in Iterative Methods for Large Linear Systems, L. Hayes and D. Kincaid, eds., San Diego, CA, 1989, Academic Press, pp. 273-291.

[6] L. C. Evans, Partial Differential Equations, American Mathematical Society, Providence, 1998.

[7] R. W. FREUND, A transpose-free quasi-minimal residual algorithm for non-Hermitian linear systems, SIAM J. Sci. Comput., 14 (1993), pp. 470-482.

[8] S. E. Howington, R. C. Berger, J. P. Hallberg, J. F. Peters, A. K. Stagg, E. W. Jenkins, and C. T. Kelley, A model to simulate the interaction between groundwater and surface water, Tech. Rep. CRSC-TR99-27, North Carolina State University, Center for Research in Scientif ic Computation, August 1999. Proceedings of the High Performance Computing Users' Group Meeting, Monterrey, CA, June 7-10.

[9] E. W. Jenkins, R. C. Berger, J. P. Hallberg, S. E. Howington, C. T. Kelley, J. H. Schmidt, A. STAGG, AND M. D. TOCCI, A two-level aggregation-based Newton-Krylov-Schwarz method for hydrol- 
ogy, in Parallel Computational Fluid Dynamics 1999, D. E. Keyes, A. Ecer, J. Periaux, and N. Satofuka, eds., North Holland, 2000, pp. xxx-yyy.

[10] - Newton-Krylov-Schwarz methods for Richards' equation, Tech. Rep. CRSC-TR99-32, North Carolina State University, Center for Research in Scientif ic Computation, October 1999. submitted for publication.

[11] C. E. KeES AND C. T. MILlER, C++ implementations of numerical methods for solving differential-algebraic equations: Design and optimization considerations, ACM Trans. Math. Soft., 25 (2000), pp. 377-403.

[12] C. T. Kelley, Iterative Methods for Linear and Nonlinear Equations, no. 16 in Frontiers in Applied Mathematics, SIAM, Philadelphia, 1995.

[13] J. MANDEL, Balancing domain decomposition, Communications in Applied Numerical Methods 9, (1993), pp. 233-241.

[14] J. MANDEL, Hybrid domain decomposition with unstructured subdomains, Contemporary Mathematics, (1994), pp. 103-112.

[15] J. Mandel AND M. BReZina, Balancing domain decomposition: Theory and performance in two and three dimensions. UCD/CCM Report 2, Nov. 1993.

[16] Y. MUALEM, A new model for predicting the hydraulic conductivity of unsaturated porous media, Water Resources Research, 12 (1976), pp. 513-522.

[17] Y. SAAD AND M. SCHULTZ, GMRES a generalized minimal residual algorithm for solving nonsymmetric linear systems, SIAM J. Sci. Stat. Comp., 7 (1986), pp. 856-869.

[18] B. Smith, P. BJøRstad, ANd W. Gropp, Domain Decomposition: Parallel Multilevel Methods for Elliptic Partial Differential Equations, Cambridge University Press, Cambridge, 1996.

[19] K. STAheli, J. H. SchmidT, AND S. SwIFT, Guidelines for Solving Groundwater Problems with ADH, January 1998.

[20] H. A. VAN DER Vorst, Bi-CGSTAB: A fast and smoothly converging variant to Bi-CG for the solution of nonsymmetric systems, SIAM J. Sci. Statist. Comput., 13 (1992), pp. 631-644.

[21] M. T. van Genuchten, Predicting the hydraulic conductivity of unsaturated soils, Soil Science Society of America Journal, 44 (1980), pp. 892-898.

[22] P. VANĔK, J. MANDEL, AND M. BREZINA, Algebraic multigrid by smoothed aggregation for second and fourth order elliptic problems, Computing, 56 (1996), pp. 179-196.

[23] J. XU, Iterative methods by space decomposition and subspace correction, SIAM Review, 34 (1992), pp. 581613.

[24] J. XU AND J. Zou, Some nonoverlapping domain decomposition methods, SIAM Review, 40 (1998), pp. 857914. 